\title{
Resistencia a medicamentos Anti-rretrovirales (ARV) en pacientes con segundo fracaso terapéutico en un programa de $\mathrm{VIH}$, Colombia
}

\author{
Anti-retroviral (ARV) drug resistance in patients with second \\ therapeutic failure in an HIV program, Colombia
}

\author{
Dagoberto Santofimio Sierra', Johanna Osorio Pinzón ${ }^{2}$, Diego Fernando Salinas Cortés², \\ Jackeline Barreto ${ }^{3}$, Jorge Andrés Ramos ${ }^{4}$, Luis Carlos Álvarez Perdomo ${ }^{5}$, Carlos Gómez A. ${ }^{5}$
}

\section{Resumen}

Objetivo: Describir la resistencia a los medicamentos antirretrovirales en pacientes con segundo fracaso terapéutico en un Programa de VIH entre enero de 2011 a Diciembre de 2011 . Material y métodos: Se realizó un estudio descriptivo, retrospectivo de serie de casos. Donde se describieron las mutaciones del VIH, características clínicas y hallazgos de laboratorio de los pacientes con resistencia en segundo fracaso terapéutico. Resultados: Se identificaron 30 pacientes con segundo fracaso terapéutico en el periodo de estudio. El tiempo promedio de uso de Terapia Anti-rretroviral por paciente fue de 77,2 meses $(D S=56$ ). A 12 pacientes se les realizaron tres cambios de esquema anti-rretroviral por fracaso terapéutico y otros 2 pacientes hasta cuatro cambios de esquema. Las mutaciones más frecuentes fueron las primarias a Inhibidores de Proteasa (M46I, I54V, L10I) y a Inhibidores de Transcriptasa Inversa Análogos de Nucleósidos (Abacavir, Didanosina y Zidovudina, Estavudina).

Palabras clave: Resistencia medicamentosa, Terapia Antirretroviral, VIH.
Abstract
Objective: to make a description of ARV drugs in patients with second therapeutic failure in an HIV Program between January and December, 2011.
Materials and methods: a descriptive, retrospective case series study was conducted. It was made a description of

HIV mutations, clinical characteristics and laboratory findings of patients with resistance in second therapeutic failure. Results: 30 patients with second therapeutic failure were identified in the study period. The average time of use of ARV therapy per patient was 77.2 (SD = 56). 12 patients underwent three changes of ARV months shema per therapeutic failure and 2 other patients up to four changes of schema. Most frequent mutations were the primary to Protease inhibitors (M46I, 154V, L101) and to inhibitors of Reverse analog Transcriptase of Nucleoside. (Abacavir, Didanosine and Zidovudine, Stavudine).

Keywords: drug resistance, ARV therapy, HIV.

\section{Introducción}

Se reporta que en 1981 se diagnosticaron los primeros pacientes con síndrome de inmunodeficiencia adquirida (SIDA) ${ }^{[1]}$ y 3 años más tarde se identificó su agente causal, el virus de la inmunodeficiencia humana $(\mathrm{VIH})^{[2]}$, posteriormente, 5 años más adelante se identificó el primer medicamento con actividad anti-rretroviral. ${ }^{[3]}$ Desde entonces, el desarrollo de la Terapia Anti-rretroviral (ARV) ha transitado cronológicamente por importantes cambios, protocolos, concentraciones para mejorar su eficacia. Sin embargo el uso de la terapia no está exenta de desarrollar resistencia. ${ }^{[4]}$

El fracaso en ARV consiste en no lograr disminuir la carga viral por debajo del limite inferior de detección, no aumentar, no aumentar el conteo de células CD4+ sobre el nivel basal o del nivel de seguridad para evitar infecciones oportunistas. ${ }^{[5]}$

Esta situación hace necesaria la realización del presente estudio donde se describe la resistencia de pacientes con segundo fracaso

1. MD, Epidemiólogo Clínico, Magister VIH SIDA, Hospital Universitario de Neiva, Colombia, Colombia.

2. MD, Internista Infectólogía, Hospital Universitario de Neiva, Colombia, Colombia.

3. MD, Control de Antibióticos, Hospital Universitario de Neiva, Neiva, Colombia.

4. Enf, Epidemiólogo, Msc (c) Epidemiología Clínica, Hospital Universitario de Neiva, Colombia.

5. MD, Internista, Hospital Universitario de Neiva, Colombia.

Datos de contacto: Dagoberto Santofimio Sierra, Correo electrónico: dagosanto@gmail.com

Recibido: 21/06/2013 - Revisado: 24/09/2013 - Aceptado: 26/06/2014 
terapéutico, las características epidemiológicas y hallazgos de laboratorio en un programa de VIH en una institución de Salud de Colombia entre enero y diciembre de 2011.

\section{Materiales y métodos}

Se realizó un estudio descriptivo, retrospectivo de series de casos el cual permite describir las mutaciones, características epidemiológicas, número de cambios de ARV, hallazgos de laboratorio y mortalidad en los pacientes en segundo fracaso terapéutico de un programa VIH de Colombia. Para la adquisición de la información fue usada la base de datos del programa en las 12 sedes en todo el país. Una vez fueron identificados los casos con más de un Fracaso Terapéutico se procede a la revisión de las historias clínicas de estos pacientes.

Fueron incluidos únicamente en el estudio los registros de pacientes con más de un fracaso terapéutico, cualquier edad y sexo, que tuvieran en sus controles reportes de carga viral (CV), recuento de Linfocitos T CD4+ y genotipo. Fueron excluidas las historias clínicas con información incompleta para el estudio (sin reportes de laboratorio necesarios para la inclusión).

Las variables estudiadas por cada paciente fueron: edad, género, Carga Viral, Recuento de Linfocitos T CD4+, numero de cambios de ARV, Perfil de resistencia. La información fue organizada en el programa de EXCEL versión 2010 e importada al programa Stata versión 8,2 para el respectivo análisis.

Se realizó estadística descriptiva; para variables cuantitativas continuas se hizo uso de la media, moda y desviación estándar. En las variables cualitativas fueron utilizados los porcentajes y frecuencias simples. Los resultados fueron presentados en tablas $\mathrm{y}$ figuras.

El estudio cumplió con los lineamientos éticos según la normatividad Colombiana Ley 23 de 1981 (Código de ética médica) y la Resolución número 8430 de 1993, emanada por el Ministerio de la Protección Social de Colombia, en la cual se determinan las normas científicas y administrativas para la investigación en salud. Según el artículo 11, este estudio se clasifica sin riesgo, la información se obtuvo a partir de las historias clínicas del archivo de instituciones que participaron en el estudio salvaguardo el secreto profesional y protección de los sujetos de los cuales no se revelan nombres ni número de historias clínicas que pudieran permitir el reconocimiento de dichos pacientes.

\section{Resultados}

Fueron encontrados 30 registros de pacientes con segundo fracaso terapéutico para el estudio. De estos, 5 de sexo femenino y 25 de sexo masculino con promedio de edad entre las mujeres fue de 37,2 años (DS=7,8) y en hombres de 43,2 años (DS=6).
En cuanto al número de cambios de la ARV se encontró que en 16 pacientes se había cambiado dos veces, en 12 pacientes en tres ocasiones mientras que a 2 pacientes habían presentado cambios en cuatro ocasiones. El tiempo promedio de ARV previo al fracaso terapéutico fue de 77,2 (DS=56) meses, con una mediana de 72 meses.

En cuanto a los hallazgos de laboratorio, los pacientes presentaron al ingreso del programa recuentos de Linfocitos T CD4+ promedio de $234(\mathrm{DS}=182)$ células $/ \mathrm{mm}^{3}$, con una mediana del 202 células $/ \mathrm{mm}^{3}$.

Respecto a los resultados de ARN carga viral VIH-1 de los pacientes al ingreso al programa, el promedio fue de 31.996 copias $/ \mathrm{ml}(\mathrm{DS}=87.724)$ y una mediana de $3.131 \mathrm{copias} / \mathrm{ml}$. A todos los pacientes se dio inicio de ARV y a las 16 semanas de terapia ARV se realizaron controles de laboratorios. El recuento promedio de Linfocitos T CD4+ posterior a la terapia ARV en los pacientes fue de 312 células $/ \mathrm{mm}^{3}(\mathrm{DS}=262$ ) con una de mediana del 258 células $/ \mathrm{mm}^{3}$; Los resultados de ARN carga viral VIH-1 posterior a la ARV fueron de 8.425 copias $/ \mathrm{ml}(\mathrm{DS}=16.131)$. A nivel general, el 30\% (9/30) de los pacientes tenían ARN carga viral VIH-1 mayor a 500 copias/ml después de 16 semanas de terapia (respuesta virológica) y el 60\% (18/30) tenían Linfocitos T CD4+ menor de 350 células $/ \mathrm{mm}^{3}$.

Los regímenes de medicamentos pertenecían a los grupos de ARV convencionales, los inhibidores de la proteasa (IP), NRTI y NNRTI. Las combinaciones más comunes fueron AZT $+3 \mathrm{TC}+\mathrm{EFV}$, $\mathrm{AZT}+3 \mathrm{TC}+\mathrm{LPV} / \mathrm{r}, \mathrm{ABC}+\mathrm{DDI}+\mathrm{LPV} / \mathrm{r}, \mathrm{AZT}+3 \mathrm{TC}+\mathrm{ATV} / \mathrm{r}$, $\mathrm{ABC}+3 \mathrm{TC}+\mathrm{LPV} / \mathrm{r}, \mathrm{AZT}+3 \mathrm{TC}+\mathrm{NVP}$. La clasificación al ingreso $\mathrm{A} 1$ $(\mathrm{N}=1), \mathrm{A} 2(\mathrm{~N}=4), \mathrm{A} 3(\mathrm{~N}=5), \mathrm{B} 1(\mathrm{~N}=1), \mathrm{B} 2(\mathrm{~N}=1), \mathrm{B} 3(\mathrm{~N}=2), \mathrm{C} 2$ $(\mathrm{N}=1), \mathrm{C} 3(\mathrm{~N}=15)$.

Las mutaciones de resistencia en el gen de la proteasa se clasifican como "mayor" o "menor". En el análisis de las mutaciones a los IP, el 30\% de los genotipos presentaron mutaciones primarias M46I, I54V, L10I. Estas mutaciones están presentes en la resistencia a ARV Atazanavir, Darunavir, Fosamprenavir, Indinavir, Lopinavir. También se presentó la mutación A71V para resistencia en Nelfinavir y en Saquinavir y la presencia de la mutación V82A muy frecuente en la mayor parte de los IP. Esta última fue la más frecuente en los genotipos tomados. De las mutaciones secundarias, las más frecuentes fueron L10I (33,3\%), L24I (23,3\%) (Tabla 1). Las posiciones $71(46,6 \%), 54(66,6 \%)$ y $10(66,3 \%)$ fueron las más prevalentes.

Otras mutaciones asociadas a genes resistentes a los IP fueron la I93L, V77I, L63P, I62V presentes en la resistencia a los siguientes ARV Atazanavir, Indinavir, Lopinavir (Tabla 2).

La mutaciones mayores M46I, para Indinavir, se presentó en 9 casos, la I54L para Darunavir se presentó en 11 casos y la V82A, mutación mayor para Lopinavir y Indinavir en 12 casos. También se presentaron mutaciones A71V, L10I/F, Q58E para resistencias menores (tabla 3 ).

Tabla 1. Mutaciones asociadas a genes de resistencia a Inhibidores de Proteasa (IP). INHIBIDORES DE PROTEASA

\begin{tabular}{|cccccccccccccccccccccccccccccc}
\hline M & A & A & A & F & G & G & I & I & I & I & I & I & K & L & L & L & L & L & L & L & L & L & Q & V & V & V & V \\
\hline 46 & 71 & 71 & 71 & 53 & 73 & 73 & 47 & 50 & 54 & 54 & 54 & 84 & 43 & 10 & 10 & 10 & 23 & 24 & 33 & 76 & 89 & 90 & 58 & 11 & 32 & 82 & 82 \\
\hline I & I & T & V & L & S & T & V & L & L & T & V & V & T & F & I & V & I & I & F & V & V & M & E & I & I & A & T
\end{tabular}

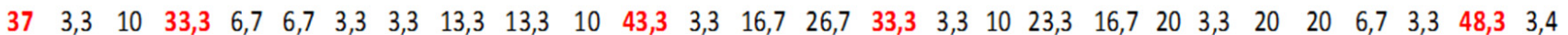


Tabla 2. Frecuencia de mutaciones asociadas con resistencia a inhibidores de proteasa (IP). OTRAS MUTACIONES DE IP

\begin{tabular}{ccccccccccccccccccccccc} 
D & I & V & E & R & R & K & R & K & M & M & L & I & L & I & I & E & V & K & L & I & I \\
\hline 60 & 93 & 82 & 35 & 57 & 57 & 20 & 41 & 20 & 36 & 36 & 63 & 15 & 89 & 85 & 93 & 34 & 77 & 77 & 33 & 13 & 62 \\
E & L & A & D & K & R & M & K & R & L & I & P & V & I & V & L & Q & I & V & I & V & V \\
6,7 & 23 & 3,3 & 13,3 & 10 & 6,7 & 6,7 & 10 & 3,3 & 17 & 3,3 & 37 & 13,3 & 3,3 & 3,3 & 6,7 & 6,7 & 20 & 6,7 & 3,3 & 3,3 & 27
\end{tabular}

Tabla 3. Mutaciones mayores y menores en genes de resistencia a inhibidores de proteasa (IP).

INHIBIDORES DE PROTEASA

\begin{tabular}{|c|c|c|c|c|c|c|c|c|c|c|c|c|c|c|c|c|c|c|c|c|c|c|c|c|}
\hline & M & A A & $\mathrm{A}$ & $\mathrm{F}$ & G & G & I & 1 & I & I & I & K & $\mathrm{L}$ & L & L L & L L & $\mathrm{L}$ & L L L & $\mathrm{L}$ & Q & V & V & V & $\mathrm{V}$ \\
\hline & 46 & 7171 & 71 & 53 & 73 & 73 & 47 & 50 & 54 & 54 & $54 \quad 84$ & $4 \quad 43$ & 10 & 10 & 1023 & 2324 & 33 & 7689 & 90 & 58 & 11 & 32 & 82 & 82 \\
\hline & I & I T & V & L & $S$ & $\mathrm{~T}$ & V & L & L & $T$ & V & $\mathrm{T}$ & $\mathrm{F}$ & I & V & | | & $\mathrm{F}$ & V V & M & $E$ & I & I & A & $\mathrm{T}$ \\
\hline Resistencia Mayor & 9 & & & & & & 1 & 3 & 4 & 2 & 11 & 2 & 2 & & & 22 & & 2 & 3 & & & 1 & 12 & 1 \\
\hline \% Resistencia Mayor & 75 & & & & & & 100 & 75 & 100 & 100 & 84,6 & 40 & 25 & & & 2928,6 & & 67 & 100 & & & 100 & 85,7 & 100 \\
\hline lesistencia Menor & 3 & 13 & 10 & 2 & 2 & 1 & & 1 & & & 1 & 1 & 6 & 10 & 1 & 55 & 5 & 11 & & 6 & 2 & & 2 & \\
\hline r Resistencia Menor & 25 & 100100 & 100 & 100 & 100 & & & 25 & & & 15,4100 & $00 \quad 60$ & 75 & 100 & & 7171,4 & 4100 & 33100 & & & 100 & & 14,3 & \\
\hline
\end{tabular}

Tabla 4. Mutaciones asociadas a genes resistentes a Inhibidores de Transcriptasa Inversa análogos de nucleósidos (NRTI).

\begin{tabular}{cccccccccccccccccccc}
\hline A & D & K & K & K & L & L & L & M & M & T & T & T & T & T & T & T & T & V & Y \\
\hline 62 & 67 & 219 & 70 & 219 & 74 & 74 & 210 & 41 & 184 & 69 & 69 & 215 & 215 & 215 & 215 & 215 & 215 & 75 & 115 \\
\hline $\mathrm{V}$ & $\mathrm{N}$ & $\mathrm{E}$ & $\mathrm{R}$ & $\mathrm{Q}$ & $\mathrm{V}$ & $\mathrm{I}$ & $\mathrm{W}$ & $\mathrm{L}$ & $\mathrm{V}$ & $\mathrm{D}$ & $\mathrm{N}$ & $\mathrm{D}$ & $\mathrm{E}$ & $\mathrm{F}$ & $\mathrm{Y}$ & $\mathrm{I}$ & $\mathrm{V}$ & $\mathrm{I}$ & $\mathrm{F}$ \\
10 & 6,7 & 10 & 16,7 & 20 & 36,7 & 3 & 23,3 & 27 & 20 & 20 & 20 & 3,3 & 27 & 6,7 & 3,3 & 20 & 6,7 & 10 & 10
\end{tabular}

Las mutaciones más frecuentes en los fármacos Inhibidores de transcriptasa reversa nucleósido (NRTI) fueron las resistentes a Abacavir, Didanosina (L74V) y zidovudina, estavudina, (M41L, D67N, L210W, T215F/Y, y K219Q).

Una mutación en la posición 215 fue la que más se presentó en los pacientes con un $67 \%$. Los anteriores patrones de mutación son consistentes para resistencia a Abacavir, Zidovudina y a Didanosina. La mutación K65R determinantes en resistencia a Lamivudina no estuvo presente en los casos (Tabla 4).

Respecto a las mutaciones de resistencias a los fármacos Inhibidores de transcriptasa reversa no nucleósido (NNRTI), las más frecuentes fueron K103N, K101Q y L100I presentes en NNRTI convencionales (Efavirenz, Nevirapina y Etravirine) (Tabla 5).

En el análisis de genotipos se comparó el perfil de resistencia del virus de acuerdo al nivel de "resistencia alta", "intermedia" y "baja”. En los IP se encontró que el 40\% tenía una resistencia alta a $\mathrm{ATV} / \mathrm{r}, \mathrm{FPV} / \mathrm{r}, \mathrm{IDV} / \mathrm{r}, \mathrm{LPV} / \mathrm{r}, \mathrm{NFV} / \mathrm{r}$, el SQV/r presento una resistencia intermedia en el $38 \%$ y el DRV/r y TPV/r fueron quienes presentaron una menor resistencia $3 \%$ ambos (Figura 1).
Tabla 5. Mutaciones asociadas a genes resistentes a Inhibidores de Transcriptasa Inversa No análogos no nucleósidos (NNRTI)

\begin{tabular}{ccccccccccc|}
\hline $\mathrm{K}$ & $\mathrm{A}$ & $\mathrm{K}$ & $\mathrm{L}$ & $\mathrm{V}$ & $\mathrm{V}$ & $\mathrm{V}$ & $\mathrm{Y}$ & $\mathrm{Y}$ & $\mathrm{Y}$ & $\mathrm{Y}$ \\
\hline 103 & 98 & 101 & 100 & 179 & 179 & 108 & 181 & 181 & 188 & 188 \\
\hline $\mathrm{N}$ & $\mathrm{G}$ & $\mathrm{Q}$ & $\mathrm{I}$ & $\mathrm{D}$ & $\mathrm{E}$ & $\mathrm{I}$ & $\mathrm{Y}$ & $\mathrm{V}$ & $\mathrm{L}$ & $\mathrm{H}$ \\
& & & & & & & & & & \\
43 & 6,7 & 13,3 & 13,3 & 10 & 3,3 & 6,7 & 3,3 & 6,7 & 6,7 & 3,3
\end{tabular}

En los NRTI se presentó una resistencia superior al $80 \%$ en FTC, $41 \%$ a DDI y $31 \%$ al AZT. Es de destacar que de los fármacos NRTI de más demanda en la prescripción para los pacientes con VIH como ABC, 3TC y AZT, las mutaciones de resistencias a estos fármacos fueron poco frecuentes a pesar de haber presentado un primer o segundo fracaso terapéutico (Figura 2).

En los NNRTI se encontró una alta resistencia en EFV 50\% y NVP 57\% (Figura 3). 


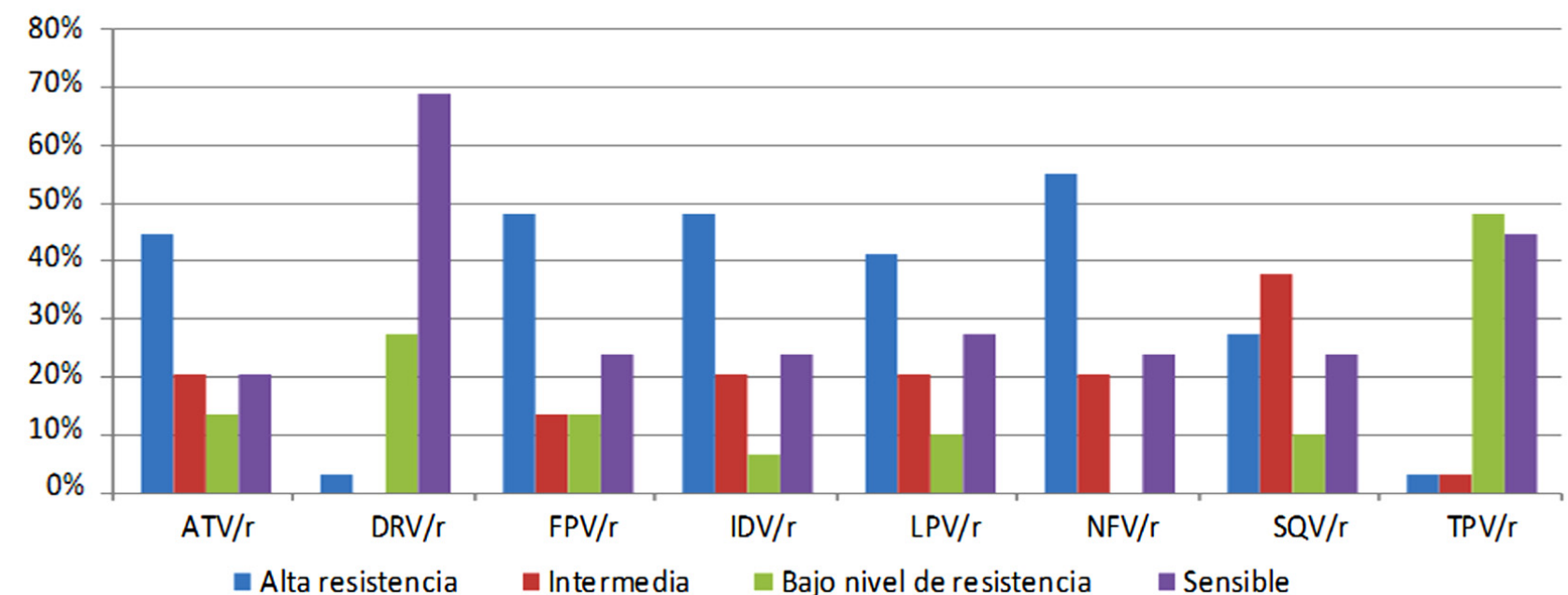

Figura 1. Perfil de resistencia a fármacos Inhibidores de Proteasa $N=30$

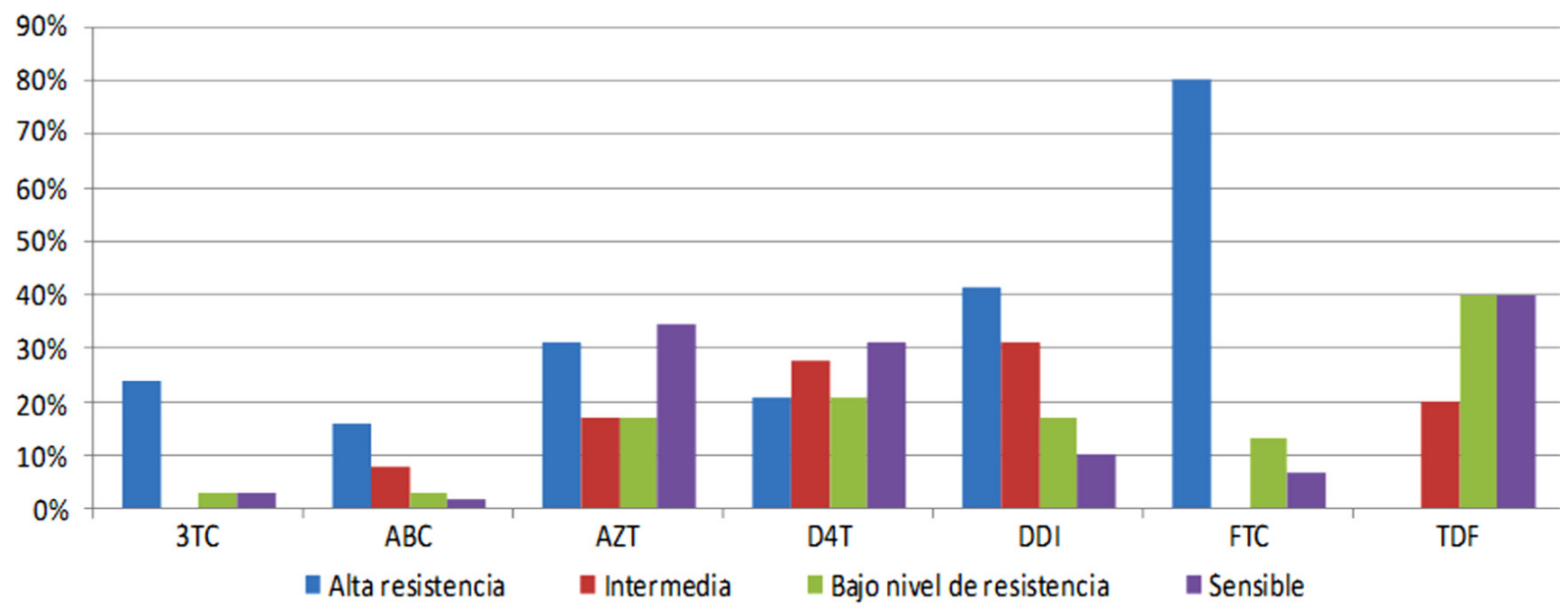

Figura 2. Perfil de Resistencia a fármacos Inhibidores de Transcriptasa Inversa análogos de nucleósidos (NRTI) N=30.

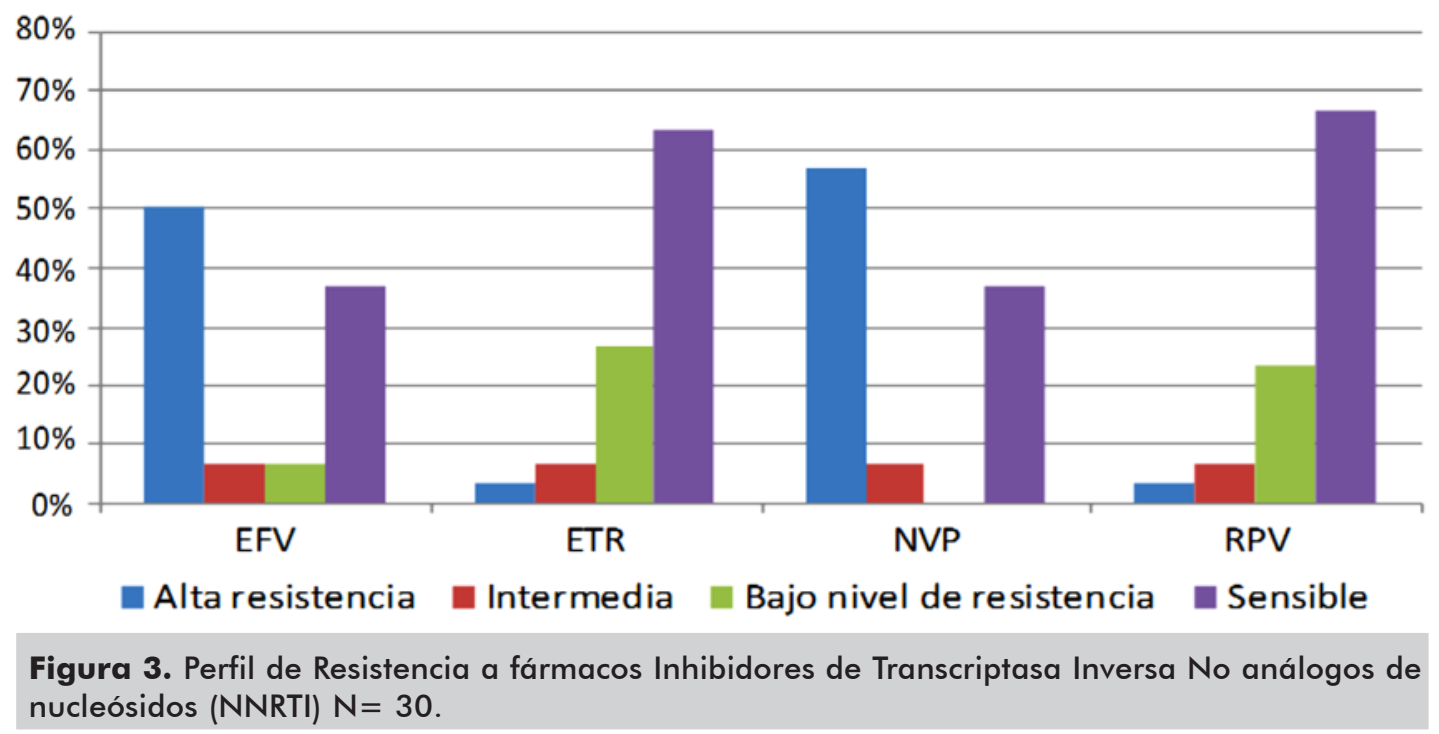




\section{Discusión}

Desde la aparición del VIH, la comunidad médica se ha preocupado por la terapia más efectiva para la infección VIH/SIDA. Sin embargo la pobre adherencia a ARV es un factor que genera problemas para que esta efectividad se cumpla. Los factores que pueden generar una pobre adherencia son los efectos tóxicos asociado a los esquemas de tratamiento y la falta de seguimiento a los pacientes en los programas. Todo lo anterior puede llevar a resistencia a los fármacos anti-rretrovirales y son las principales barreras para lograr una supresión mantenida del virus durante la terapia. ${ }^{[6]}$

El aumento de la resistencia obedece a la transmisión de virus resistente a fármacos anti-rretrovirales. Algunos inhibidores de trancriptasa inversa (NRTI) nucleósidos (o nucleótidos) presentan mutaciones como la T215Y y H208Y, las cuales dan hipersensibilidad viral los no análogos de nuclesidos (NNRTI) incluyendo la etravirina. ${ }^{[6]}$ En nuestro estudio las resistencias a fármacos NNRTI fueron menores, solo un 3,3\% de los individuos tenían la presencia de estas mutaciones principalmente a fármacos ARV Etravirine y Rilpivirine. De acuerdo con la literatura, el complejo de inserción 69 está asociado con la resistencia a todos los NRTI. Este trabajo mostró las mutaciones T69N (20\%) y T69D (20\%) en los genotipos tomados a pacientes del programa. No se presentó en ninguno de los genotipos el complejo de mutación Q151M susceptible a Tenofovir.

Las mutaciones que se conocen como Análogos de Timidina (TAMs) (M41L, D67N, K70R, L210W, T215Y/F, y K219Q/E) todas fueron expresadas en los genotipos de los pacientes siendo más frecuente la presencia de la M41L. Estas mutaciones confieren una susceptibilidad reducida a todos los aprobados NRTI sin embargo este grado depende de la resistencia cruzada y el número de mutaciones involucradas. ${ }^{[6,7]}$ Las mutaciones de la transcriptasa inversa E44D y V118I no fueron presentes en los genotipos de este estudio. Estas mutaciones tienen un papel adyuvante en el aumento de la resistencia al NRTI con presencia de TAMs. La mutación M184V fue presente en el 20\% de los genotipos. Esta mutación aumenta la resistencia al Abacavir cuando se asocia a los TAMs al igual que la mutación K65R con el Tenofovir. Finalmente la frecuencia de TAMs en nuestro estudio fue baja respecto a lo que se reporta en la literatura.

La Didanosina presento una resistencia alta, superior del $40 \%$, la presencia en al menos tres de las siguientes mutaciones M41L, D67N, L210W, T215Y/F, K219Q/E hace la asociación de la resistencia a Didanosina. ${ }^{[6]}$

Las sustituciones $\mathrm{T} 215 / \mathrm{D} / \mathrm{E} / \mathrm{F} / \mathrm{Y} / \mathrm{I} / \mathrm{V}$ son mutaciones en el codón 215 revertientes que le confieren un mayor riesgo de fracaso virológico frente a Zidovudina o Estavudina. ${ }^{[6]}$ En nuestro estudio, la presencia de este complejo fue el más frecuente en los genotipos tomados. Sin embargo la resistencia a ambos fármacos no supero el $30 \%$. Esta mutación T215Y puede surgir rápidamente de una de estas mutaciones en el presencia de Zidovudina o Estavudine.

El Tenofovir no mostro resistencia alta del virus. Esto podría estar implicado por la ausencia de K65R que está asociada con una disminución de la respuesta virológica. De acuerdo con la literatura, la presencia de TAMs o combinado el tratamiento con Zidovudina previene la aparición de K65R en presencia de Tenofovir. ${ }^{[6]}$

En los NNRTI, la Etravirina evidenció una resistencia muy baja (3,3\%). La resistencia a Etravirina ha sido extensamente estudiada en genotipos sólo en el contexto de la coadministración ${ }^{[5]}$. Este punto de corte permite tener una cierta confianza en el manejo del VIH con el fármaco.
La Rilpivirina al igual que la Etravirina presentó una menor frecuencia de resistencia ARV. La causa está relacionada por la ausencia de la combinación de E138K y M184I que demostraron 6,7 veces más reducida susceptibilidad fenotípica a rilpivirina y con 2,8 veces la reducción de $\mathrm{E} 138 \mathrm{~K}^{[5]}$.

En los IP, la mutación mayor de resistencia más frecuente fue la M46I. La unión con la mutación L76V podría aumentar susceptibilidad a Atazanavir. ${ }^{[6]}$ Las mutaciones de la proteasa I47V, I84V fueron identificadas en los pacientes del estudio; las mutaciones (I54M, T74P) no se encontraron en los genotipos aplicados. Los anteriores genes tienen un impacto negativo sobre los IP. Contrario a lo anterior, la V82A se presentó en un 3\%. Esta última produce un impacto positivo sobre la respuesta virológica a Darunavir/ Ritonavir.

En el análisis de las mutaciones primarias encontradas en los IP fueron M46I, I54V, L10I, presentes en resistencia a Atazanavir, Darunavir, Fosamprenavir, Indinavir, Lopinavir y la V82A fue la más frecuente en la mayor parte de los IP, fue la más frecuente en los genotipos tomados $48,3 \%$. Las mutaciones específicas, lo más notablemente I47V y V32I, se asocian con alto nivel resistencia. ${ }^{[5]}$ La presencia de L76V (20\%) asociada a la resistencia aumenta sustancialmente la resistencia a Lopinavir/Ritonavir. ${ }^{[6]}$

En Colombia de acuerdo a las guías de atención de VIH/SIDA del año 2006 no se contempla la solicitud de rutina de genotipos previos al inicio de la ARV.

En otros países latinoamericanos como Argentina se ha encontrado una prevalencia de resistencia a fármacos ARV entre $2,4 \mathrm{y}$ $15,4 \%$, con una frecuencia de mutaciones secundarias de hasta $90 \%$. Estudios, en Brasil, han demostrado una prevalencia de hasta 2,2\% de resistencia a IP, $2,4 \%$ a ITRN y $2,1 \%$ a NNRTI. ${ }^{7}$ Las mutaciones reportadas más frecuentemente en los trabajos mencionados, poco difieren a las encontradas por nuestro trabajo, la V82A, la A71V muy frecuente. En los IP. La L74V T215E la más frecuente para los NRTI y K103N para los NNRTI. [7]

Sería de gran interés ampliar el grupo de estudio y complementar la información con datos del tiempo desde el contagio. Además, realizar un seguimiento de los pacientes sería muy interesante, ya que al contar con un estudio de genotipificación previo al inicio de terapia ARV, se podría evaluar los cambios en el perfil de resistencia viral determinados por el uso de drogas anti-rretrovirales. ${ }^{[7]}$

\section{Conclusiones}

Las mutaciones encontradas fueron a los IP siendo mutaciones primarias M46I, I54V, L10I presente en resistencia a Atazanavir, Darunavir, Fosamprenavir, Indinavir, Lopinavir y la V82A fue la más frecuente. En los fármacos NRTI fueron para Abacavir, Didanosina y Zidovudina, Estavudina. Estos resultados demuestran que los perfiles de resistencia son similares a los documentados en la literatura. Los pacientes candidatos a genotipo fueron pacientes dos o más fracasos, no hubo pacientes no tratados previamente, debido a los protocolos del programa no fueron candidatos. Es relevante destacar que la mayor parte de los fracasos terapéuticos estudiados fueron debidos a la adquisición de mutaciones de resistencia, la alta adherencia al tratamiento disminuye la probabilidad de fracaso en los pacientes del programa. Sería interesante para próximos estudio aplicar genotipos a pacientes "Naive" o sin tratamiento anti-rretroviral previo para identificar posible resistencia en este grupo de pacientes y poder optimizar así los regímenes de antirretrovirales. 


\section{Agradecimiento}

Al programa SIES Salud y al personal administrativo y asistencial de todas las Sedes que pusieron a disposición la información para llevar a cabo este proyecto.

\section{Bibliografía}

1. Pneumocystis pneumonia - Los Angeles. MMWR Morb Mortal Wkly Rep, 1981. 30(21):250-2.

2. Barre-Sinoussi, F., et al., Isolation of a T-lymphotropic retrovirus from a patient at risk for acquired immune deficiency syndrome (AIDS). Science, 1983. 220(4599): 868-71.

3. Fischl, M.A., et al., The efficacy of azidothymidine (AZT) in the treatment of patients with AIDS and AIDS-related complex. A double-blind, placebo-controlled trial. N Engl J Med, 1987. 317(4):185-91.

4. Metzner, K.J., et al., Minority quasispecies of drugresistant HIV- 1 that lead to early therapy failure in treatment-naive and-adherent patients. Clin Infect Dis, 2009. 48(2):239-47.

5. Erice A, M.S., Gatell JM. Significado clínico de la resistencia del VIH a los anti-rretrovirales, in Resistencia del $\mathrm{VIH}$ a los fármacos anti-rretrovirales. E. Antares, Editor. Barcelona. 1999. 123-131.

6. Afani S, A., et al., Resistencia primaria a terapia antirretroviral en pacientes con infección por VIH/SIDA en Chile. Revista médica de Chile, 2005. 133:295-301.

7. Kuritzkes, D.R., et al., Rate of thymidine analogue resistance mutation accumulation with zidovudine- or stavudine-based regimens. J Acquir Immune Defic Syndr, 2004. 36(1):600-3. 